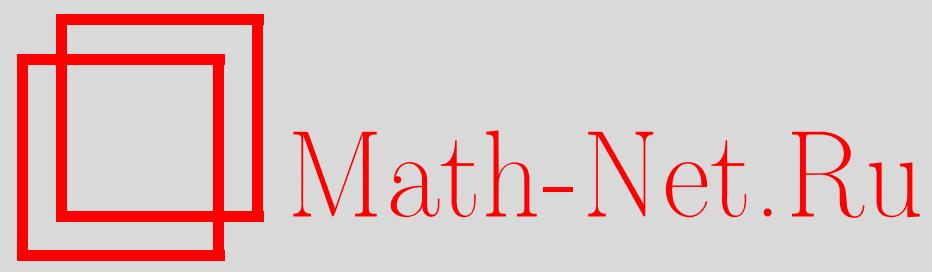

А. А. Туганбаев, Порядки в цепных кольцах, Матем. заметки, 2003, том 74, выпуск 6, 924-933

DOI: https://doi.org/10.4213/mzm320

Использование Общероссийского математического портала Math-Net.Ru подразумевает, что вы прочитали и согласны с пользовательским соглашением http://www . mathnet.ru/rus/agreement

Параметры загрузки:

IP: 18.234 .156 .22

26 апреля 2023 г., 03:02:50

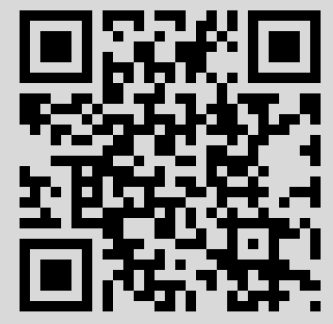




\title{
ПОРЯДКИ В ЦЕПНЫХ КОЛЬЦАХ
}

\section{А. А. Туганбаев}

\begin{abstract}
Пусть $A$ - кольцо и $T(A)$ и $N(A)$ - множество всех регулярных элементов и множество всех нерегулярных элементов кольца $A$ соответственно. Доказано, что $A$-правый порядок в цепном справа кольце тогда и только тогда, когда множество всех регулярных элементов кольца $A$ является левьп идеалом мультипликативной полугруппы $A$ и для любых элементов $a_{1}$ и $a_{2}$ кольца $A$ либо существуют такие элементы $b_{1} \in A$ и $t_{1} \in T(A)$, что $a_{1} b_{1}=a_{2} t_{1}$, либо существуют такие элементы $b_{2} \in A$ и $t_{2} \in T(A)$, что $a_{2} b_{2}=a_{1} t_{2}$. Дистрибутивное справа кольцо $A$ является правым порядком в цепном справа кольце тогда и только тогда, когда множество $N(A)$ является левым идеалом кольца $A$. Если $A$ - такое дистрибутивное справа кольцо, что все его правые делители нуля содержатся в радикале Джекобсона $J(A)$ кольца $A$, то $A$ - правый порядок в цепном справа кольце.
\end{abstract}

Библиография: 11 названий.

В данной работе все кольца предполагаются ассоциативными и с ненулевой единицей. Модуль $M$ называется иепныц, если любые два его подмодуля сравнимы по включению, т.е. решетка Lat $(M)$ всех его подмодулей является цепью (термин введен Л. А. Скорняковым). Цепные справа кольца подробно исследуются в книгах Дубровина, Бессенродта, Брунгса и Тёнера, (см. [1]-[3]); кроме того, следует отметить работы Дубровина [4], [5]. Модуль $M$ назьвается дистрибутивнылм, если решетка $\operatorname{Lat}(M)$ дистрибутивна. Дистрибутивные модули и кольца исследуются в книгах Беренса, Кона и Туганбаева (см. [6]-[9]). Кольцо без делителей нуля называется областью. Радикал Джекобсона и множество всех правых или левых делителей нуля кольца $A$ обозначаются через $J(A)$ и $N(A)$ соответственно; множество $A \backslash N(A)$ обозначается через $T(A)$, а элементы множества $T(A)$ называются регулярныц ми әлементами (в $A)$. Мультипликативное замкнутое подмножество $T$ множества $T(A)$ называется правым. множеством Оре, если выполняются следуюшие два эквивалентных условия:

(1) для любых элементов $a \in A$ и $t \in T$ сушествуют такие элементы $b \in A$ и $u \in T$, что $a u=t b$

(2) существует такое кольцо $A_{T}$, что $A$ - унитарное подкольцо в $A_{T}$, все элементы множества $T$ обратимы в $A_{T}$ и $A_{T}=\left\{a t^{-1} \mid a \in A, t \in T\right\}$.

В этом случае кольцо $A_{T}$ называется правым кольиом частных кольца $A$ относительно $T$. Если $T(A)$ - правое множество Оре, то кольцо $A_{T(A)}$ называется правылм

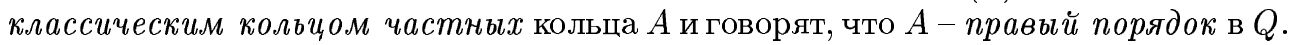

Работаподлержана Российским фондом фундаментальных исследований, проект № 99-01-00382. 
Хорошо известно, что область $A$ является правым порядком в теле тогда и только тогда, когда $T(A)$ - правое множество Оре. Кроме того, любая дистрибутивная справа область является правым порядком в теле [10]. Заметим, что каждое тело является цепным кольцом. Основными результатами данной работы являются теоремы 1-3.

ТЕОрема 1. Кольцо А является правым порядком в иепном справа кольце тогда и только тогда, когда множсество $N(A)$ является левым идеалом мультипликативной полугруппь $A$ и для любых әлементов $a_{1}$ и $a_{2}$ кольиа $A$ либо существуют такие әлементы $b_{1} \in A u t_{1} \in T(A)$, что $a_{1} b_{1}=a_{2} t_{1}$, либо существуют такие әлементы $b_{2} \in A u t_{2} \in T(A)$, что $a_{2} b_{2}=a_{1} t_{2}$.

Отметим связь нашей теоремы 1 с классической теоремой Оре о правых порядках в телах. Кроме того, теорема 1 используется для доказательства теорем 1 и 2.

Теорема 2. Дистрибутивное справа кольцо А является правым порядком в иепном справа кольче тогда и только тогда, когда множество $N(A)$ является левьм идеалом кольиа $A$.

Теорема 3. Если $A$ - такое дистрибутивное справа кольцо, что все его правые делители нуля содержатся в радикале Джекобсона $J(A)$ кольиа $A$, то $A$ - правый порядок в цепном справа кольце.

Доказательства теорем 1, 2 и 3 разбиты на ряд утверждений. Приведем необходимые обозначения и определения. Кольцо $A$ назьвается локальным, если фактор-кольцо $A / J(A)$ является телом (это равносильно тому, что для любого необратимого элемента $a \in A$ элемент $1-a$ обратим). Непосредственно проверяется, что все цепные справа кольца локальны. Если $A$ - кольцо и $M$ - правый (соответственно левьй) $A$-модуль, то через $N_{r}(M)$ (соответственно $N_{\ell}(M)$ ) обозначается множество всех элементов кольца $A$ аннулирующих ненулевые элементы модуля $M$; в частности, $N_{r}(A)$ и $N_{\ell}(A)$ - множество всех правых делителей нуля и множество всех левых делителей нуля кольца $A$ соответственно. Ясно, что $N(A)=N_{r}(A) \cup N_{\ell}(A)$.

Модуль называется равномерным, если любые два его ненулевых подмодуля имеют ненулевоепересечение. Подмодуль $N$ модуля $M$ назьвается существенным (в $M)$, если $N \cap X \neq 0$ для каждого ненулевого подмодуля $X$ модуля $M$. Через $Z\left(A_{A}\right)$ обозначается множество всех элементов кольца $A$, правые аннуляторы которых являются существенными правыми идеалами кольца $A$. Хорошо известно, что $Z\left(A_{A}\right)$ - идеал кольца $A$ (см., например, утверждение 1.28 из [10]); этот идеал называется правым сингулярным идеалом кольца $A$. Правьй или левый идеал $B$ кольца $A$ называется вполне первичным, если $B \neq A$ и множество $A \backslash B$ мультипликативно замкнуто.

ПРЕДЛОЖЕНИЕ 1. Пусть $A-$ кольцо, $T=T(A)$ u $N=N(A)$. Тогда равносильнь следующие условия:

(1) $A$ - правый порядок в локальном кольче $Q$;

(2) $T$ - правое множество Оре и $N$ - подполугруппа аддитивной группь $A$;

(3) $A$ - правый порядок в локальном кольце $Q$, радикал Джекобсона $J(Q)$ кольиа $Q$ совпадает с множеством $\bar{N}$ всех нерегулярных әлементов кольиа $Q$ и $N$ вполне первичный идеал кольиа $A$, совпадающий с множеством $A \cap J(Q)$.

ДоказАТЕльство. $(1) \Rightarrow(2)$ Так как $A$ имеет правое классическое кольцо частных, то $T$ - правое множество Оре. Пусть $a$ и $b$ - нерегулярные элементы кольца $A$. Тогда $a$ 
и $b$ - необратимые элементы кольца $Q$. Так как кольцо $Q$ локально, то элемент $a+b$ не обратим в $Q$. Поэтому элемент $a+b$ не регулярен в $A$ и $N-$ подполугруппа аддитивной группы $A$.

$(2) \Rightarrow(3)$ Так как $T$ - правое множество Оре, то кольцо $A$ имеет правое классическое кольцо частных $Q$. Пусть $q$ - необратимьй элемент кольца $Q$. Существуют такие элементы $a \in A$ и $t \in T$, что $q=a t^{-1}$. Так как элемент $q$ не обратим в $Q$, то элемент $a$ не регулярен в $A$. Так как $N$ - подполугруппа аддитивной группы $A$ и сумма нерегулярного элемента $a \in A$ и элемента $t-a$ является регулярным элементом $t$ кольца $A$, то элемент $t-a$ регулярен в $A$. Поэтому элемент $t-a$ обратим в $Q$. Так как $1-q=1-a t^{-1}=(t-a) t^{-1}$, то элемент $1-q$ обратим в $Q$. Поэтому кольцо $Q$ локально.

Так как $Q$ - локальное кольцо и все регулярные элементы кольца $Q$ обратимы в $Q$, то $J(Q)=\bar{N}$. Так как $J(Q)$ - вполне первичньй идеал кольца $Q$, то $A \cap J(Q)$ - вполне первичный идеал кольца $A$. Так как

$$
N \subseteq A \cap \bar{N}=A \cap J(Q)
$$

то остается доказать, что $A \cap J(Q) \subseteq N$. Пусть $a$ - элемент идеала $A \cap J(Q)$. Элемент $a$ не обратим в $Q$. Поэтому элемент $a$ не регулярен в $A$ и $a \in N$.

Импликация $(3) \Rightarrow(1)$ очевидна.

Для удобства следующие три известные леммы мы приведем с краткими доказательствами.

Лемма 1 [11]. Пусть $A-$ кольцо $и M-$ правый $A$-модуль. Тогда равносильньь следующие условия:

(1) $M-$ - истрибутивный модуль;

(2) для любых әлементов $x$ и у модуля $M$ существует такой элемент а кольиа $A$, что әлементы ха и $y(1-a)$ содержстая в пересечении модулей $x A$ и у

ДокАЗАТЕЛЬСТво. $(1) \Rightarrow(2)$ Так как $M-$ дистрибутивньй модуль, то

$$
(x+y) A=(x+y) A \cap(x A+y A)=(x+y) A \cap x A+(x+y) A \cap y A .
$$

Поэтому существуют такие элементы $a$ и $b$ кольца $A$, что

$$
x+y=(x+y) b+(x+y) a, \quad(x+y) b \in x A \quad \text { и } \quad(x+y) a \in y A .
$$

Тогда

$$
y b=(x+y) b-x b \in x A \cap y A \quad \text { и } x a=(x+y) a-y a \in x A \cap y A .
$$

Tак как $(x+y)(1-a-b)=0$, то

$$
y(1-a)=(x+y)(1-a-b)-x(1-a-b)+y b=x(a+b-1)+y b \in x A \cap y A .
$$

Поэтому $a$ - требуемьй элемент кольца $A$.

$(2) \Rightarrow(1)$ Пусть $X, Y$ и $Z$ - подмодули модуля $M$ и $z$ - элемент модуля $(X+Y) \cap Z$. Сушествуют такие элементы $x \in X$ и $y \in Y$, что $z=x+y$. По условию сушествует 
такой элемент $a$ кольца $A$, что элементы $x a$ и $y(1-a)$ содержатся в модуле $x A \cap y A$. Тогда

$$
\begin{gathered}
z(1-a)=x(1-a)+y(1-a) \in x A \cap z A \in X \cap Z \\
z a=x a+y a \in y A \cap z A \in Y \cap Z \\
z=z(1-a)+z a \in X \cap Z+Y \cap Z \\
(X+Y) \cap Z \subseteq X \cap Z+Y \cap Z \subseteq(X+Y) \cap Z
\end{gathered}
$$

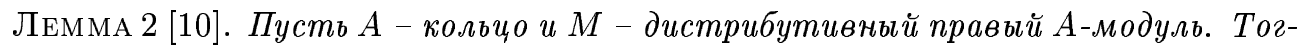
да верны следующие утверэсдения:

(1) если х и $y$-такие әлементы модуля $M$, что $x A \cap y A=0$, то существует такой әлемент а кольиа $A$, что $x a=0$ и $y(1-a)=0$;

(2) если $X$ и $Y$ - такие подмодули модуля $M$, что $X \cap Y=0, \operatorname{mo} \operatorname{Hom}(X, Y)=$ $\operatorname{Hom}(Y, X)=0$

(3) для каждого равномерного правого $A$-модуля $N$ и любых гомоморфизмов $f, g: M \rightarrow N$ подмодули $\operatorname{Ker}(f)$ u $\operatorname{Ker}(g)$ модуля $M$ сравнимы по включению;

(4) если $A$ - равномерное справа кольцо, то $M$ - равномерный модуль тогда и только тогда, когда для любых әлементов $x$ и у модуля $M$ правые аннуляторы $r(x)$ и $r(y)$ сравнимы по включению;

(5) если $A$ - дистрибутивное справа кольцо, то для любих двух вполне первичных правых идеалов $X$ и $Y$ кольиа $A$ верно, что либо $X+Y=A$, либо правые идеалы $X$ и $Y$ сравнимы по включению.

ДоКАЗАТЕЛЬСТво. (1) следует из леммы 1.

(2) Пусть $f: x A \rightarrow y A$ - гомоморфизм, $x$ - элемент модуля $X$ и $y \equiv f(x) \in Y$. По (1) существует такой элемент $a$ кольца $A$, что $x a=0$ и $y(1-a)=0$. Тогда $y=y a+y(1-a)=$ $y a=f(x a)=f(0)=0$. Поэтому $\operatorname{Hom}(X, Y)=0$.

(3) Допустим противное. Существуют ненулевые элементы $u \in \operatorname{Ker}(g) \backslash \operatorname{Ker}(f)$ и $v \in \operatorname{Ker}(f) \backslash \operatorname{Ker}(g)$. Ненулевые подмодули $f(u A)$ и $g(v A)$ равномерного модуля $N$ имеют ненулевое пересечение. Поэтому существует ненулевой элемент $t \in f(u A) \cap g(v A)$. Существуют такие элементы

$$
x \in u A \subseteq \operatorname{Ker}(g) \quad \text { и } \quad y \in v A \subseteq \operatorname{Ker}(f),
$$

что

$$
t=f(x)=g(y) \neq 0 .
$$

По лемме 1 существует такой элемент $a$ кольца $A$, что элементы $x a$ и $y(1-a)$ содержатся в модуле $x A \cap y A \subseteq \operatorname{Ker}(f) \cap \operatorname{Ker}(g)$. Поэтому $t a=f(x a)=0$ и $t(1-a)=g(x(1-a))=0$. Так как $0 \neq t=t a+t(1-a)=0$, то получаем противоречие.

(4) Докажем импликацию $\Leftarrow$. Пусть $x$ и $y$ - такие элементы модуля $M$, что $x A \cap y A=0$. По условию можно считать, что $r(x) \subseteq r(y)$. По (1) существует такой элемент $a$ кольца $A$, что $a \in r(x) \subseteq r(y)$ и $(1-a) \in r(y)$. Тогда $1=a+(1-a) \subseteq r(y)$. Поэтому $y=0$ и $M$ - равномерный модуль.

$\Rightarrow$ Существуют такие гомоморфизмы $f, g: A_{A} \rightarrow M$, что $f(1)=x$ и $g(1)=y$. По (3) можно считать, что $\operatorname{Ker}(f) \subseteq \operatorname{Ker}(g)$. Так как $\operatorname{Ker}(f)=r(x)$ и $\operatorname{Ker}(g)=r(y)$, то $r(x) \subseteq$ $r(y)$. 
(5) Допустим, что правые идеалы $X$ и $Y$ не сравнимы по включению. Тогда существуют элементы $x \in X \backslash Y$ и $y \in Y \backslash X$. По лемме 1 существует такой элемент $a$ кольца $A$, что $x a \in y A \subseteq Y$ и $y(1-a) \in x A \subseteq X$. Так как правые идеалы $X$ и $Y$ вполне первичны, то $a \in Y$ и $1-a \in X$. Поэтому

$$
A=(1-a) A+a A \subseteq X+Y \subseteq A .
$$

Лемма 3. (1) Если $A$ - равномерное справа кольцо и $N_{\ell}$ - множсество всех левых делителей нуля кольиа $A$, то $N_{\ell}$ - вполне первичный идеал кольца $A$, совпадающий с правым сингулярным идеалом $Z\left(A_{A}\right)$ кольиа $A$.

(2) Если $X$ - вполне первичный правый идеал кольиа $A$, содержсащийся в $J(A)$, то $X-$ идеал кольиа $A$.

ДокАЗАтЕльство. (1) Так как $A$ - равномерное справа кольцо, то каждьй ненулевой правьй идеал кольца $A$ является существенным. Поэтому $N_{\ell}(A)$ совпадает с правым сингулярным идеалом $Z\left(A_{A}\right)$ кольца $A$. В частности, $N_{\ell}(A)$ - идеал кольца $A$. Непосредственно проверяется, что $N_{\ell}(A)$ - вполне первичньй идеал.

(2) Пусть $x$ - элемент правого идеала $X$ и $a$-элемент кольца $A$. Элемент $1+a x a$ обратим в $A$, обратимый элемент $(1+a x a)^{-1}$ не содержится во вполне первичном правом идеале $X$ и $(1+a x a)^{-1}(1+a x a) x=x \in X$. Поэтому

$$
(1+a x a) x \in X \quad \text { и } \quad(a x)^{2}=(1+a x a) x-x \in X .
$$

Так как $(a x)^{2} \in X$ и $X-$ вполне первичньй правьй идеал, то $a x \in X$ и $X$ - идеал кольца $A$.

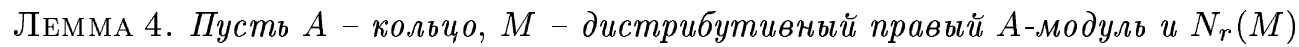
- множество всех әлементов кольца $A$, являюшихся правыми аннуляторами ненулевых әлементов модуля $M$. Тогда верны следующие утверэсдения:

(1) для любых двух множеств $\left\{X_{i}\right\}_{i \in I} u\left\{Y_{j}\right\}_{j \in J}$ подмодулей модуля $M$ выполнено равенство

$$
\left(\sum_{i \in I} X_{i}\right) \cap\left(\sum_{j \in J} Y_{j}\right)=\sum_{i \in I, j \in J}\left(X_{i} \cap Y_{j}\right) \text {; }
$$

(2) если $X$ и $Y$ - такие подмодули модуля $M$, что $X \cap Y=0$, то существуют такие вполне инвариантные подмодули $\bar{X}$ и $\bar{Y}$ модуля $M$, что

$$
X \subseteq \bar{X}, \quad Y \subseteq \bar{Y}, \quad \operatorname{Hom}(X, Y)=\operatorname{Hom}(Y, X)=0
$$

(следовательно, $M$ - равномерный модуль тогда и только тогда, когда любые два его вполне инвариантных ненулевых подмодуля имеют ненулевое пересечение);

(3) если $1-a \notin N_{r}(M)$ для каждого әлемента $a \in N_{r}(M)$, то $M-$ равномерный модуль;

(4) если $N_{r}(M)$ - подполугруппа аддитивной группы $A$, то $M$ - равномерный модуль;

(5) если $N_{r}(M) \subseteq J(A)$, то $M-$ равномерный модуль;

(6) если $A$ - равномерное справа кольчо, то следующие три условия әквивалентны:

а) $M-$ равномерный модуль;

б) $N_{r}(M)-$ подполугруппа аддитивной группьи $A$;

в) $N_{r}(M)$ - вполне первичный правый идеал кольиа $A$; 
(7) если $A$ - равномерное справа кольцо и $N_{r}(M) \subseteq J(A)$, то $M-$ равномерный модуль и $N_{r}(M)$ - вполне первичный идеал кольиа $A$.

ДокАЗАТЕЛьСТво. (1) проверяется непосредственно.

$(2)$ Обозначим через $\bar{X}$ и $\bar{Y}$ вполне инвариантные подмодули $\sum_{g \in \operatorname{End}(M)} g(X)$ и $\sum_{h \in \operatorname{End}(M)} h(Y)$ модуля $M$ соответственно. С использованием (1) и леммы $2(2)$ непосредственно проверяется, что $\bar{X} \cap \bar{Y}=0$.

(3) Пусть $x$ - ненулевой элемент модуля $M$ и $y$ - такой элемент модуля $M$, что $x A \cap y A=0$. По лемме $2(1)$ существует такой элемент $a$ кольца $A$, что $x a=0$ и $y(1-a)=0$. Тогда $a \in N_{r}(M)$. По условию $1-a \notin N_{r}(M)$. Тогда $y=0$, поскольку $y(1-a)=0$. Поэтому $M-$ равномерньй модуль.

(4) Пусть $a$ - элемент множества $N_{r}(M)$. По (3) достаточно доказать, что $1-a \notin$ $N_{r}(M)$. Допустим противное. Так как $N_{r}(M)$ - подполугруппа аддитивной группы $A$, то $1=a+(1-a) \in N_{r}(M)$. Поэтому $m \cdot 1=0$ для некоторого ненулевого элемента $m$ модуля $M ;$ получено противоречие.

(5) Пусть $a$ - элемент множества $N_{r}(M) \subseteq J(A)$. Тогда элемент $1-a$ обратим в $A$. Поэтому $1-a \notin N_{r}(M)$. По (3) $M$ - равномерный модуль.

(6) Докажем первую эквивалентность. Импликация б) $\Rightarrow$ а) следует из (4).

а) $\Rightarrow б)$ Пусть $b$ и $c$ - элементы множества $N_{r}(M)$. Существуют такие ненулевые элементы $x$ и $y$ модуля $M$, что $b \in r(x)$ и $c \in r(y)$. По лемме $2(4)$ правые идеалы $r(x)$ и $r(y)$ сравнимы по включению. Без ограничения общности можно считать, что $r(x) \subseteq r(y)$. Тогда $b+c \in r(x)+r(y)=r(y)$. Поэтому $b+c \in N_{r}(M)$.

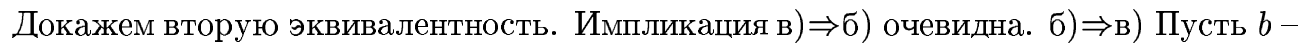
элемент множества $N_{r}(M)$. Существует такой ненулевой элемент $x$ модуля $M$, что $x b=0$. Так как $b A \subseteq r(x)$ и $N_{r}(M)$ - подполугрупша аддитивной групшы $A$, то $N_{r}(M)$ - правьй идеал кольца $A$.

Допустим, что $b=c d$ для некоторых элементов $c$ и $d$ кольца $A$ и $c \notin N_{r}(M)$. Тогда $x c \neq 0$ и $(x c) d=x b=0$. Поэтому $d \in N_{r}(M)$ и $N_{r}(M)$ - вполне первичньй правьй идеал.

(7) По (5) $M$ - равномерньй модуль. По (6) $N_{r}(M)$ - вполне первичньй правьй идеал кольца $A$. По лемме $3(2) N_{r}(M)$ - идеал кольца $A$.

ПРЕДЛОЖЕНИЕ 2. Пусть $A-\kappa о л ь и о, ~ T=T(A), N=N(A) u N_{\ell}=N_{\ell}(A)$. Тољдa равносильны следующие условия:

(1) $A$ - правый порядок в чепном справа кольие $Q$;

(2) $N$ - левый идеал мультипликативной полугруппы $A$ и для любых әлементов $a_{1}$ и $a_{2}$ кольиа $A$ либо существуют такие әлементы $b_{1} \in A u t_{1} \in T$, что $a_{1} b_{1}=$ $a_{2} t_{1}$, либо существуют такие әлементы $b_{2} \in A u t_{2} \in T$, что $a_{2} b_{2}=a_{1} t_{2}$;

(3) $A$ - равносильное справа кольчо, являющееся правым порядком в чепном справа кольие $Q$, радикал Джекобсона $J(Q)$ кольиа $Q$ совпадает смножеством $\bar{N}$ всех нерегулярных әлементов кольца $Q, N$ - вполне первичный идеал кольца $A$, совпадающий с множеством $A \cap J(Q), N_{\ell}$ - вполне первичный идеал кольца $A$, совпадающий с правым сингулярным идеалом $Z\left(A_{A}\right)$ кольиа $A$, множсество $\bar{N}_{r}$ всех правых делителей нуля кольиа $Q$ является вполне первичным идеалом в $Q$, множество $\bar{N}_{\ell}$ всех левых делителей нуля кольиа $Q$ является вполне первичным 
идеалом в $Q$, совпадающим с правым сингулярным идеалом $Z\left(Q_{Q}\right)$ кольиа $Q, u$ $\bar{N}_{\ell}=N_{\ell} Q$.

ДоказАТЕльСтво. (1) $\Rightarrow(2)$ По предложению $1 N$ - идеал кольца $A$. Пусть $a_{1}$ и $a_{2}-$ элементы кольца $A$. Так как $A$ - правьй порядок в цепном справа кольце $Q$, то либо существуют такие элементы $b_{1} \in A$ и $t_{1} \in T$, что $a_{1} b_{1} t_{1}^{-1}=a_{2}$, либо существуют такие элементы $b_{2} \in A$ и $t_{2} \in T$, что $a_{2} b_{2} t_{2}^{-1}=a_{1}$. Поэтому выполняется условие (2).

$(2) \Rightarrow(3)$ Докажем, что $A$ имеет правое классическое кольцо частных $Q$. Достаточно доказать, что $a T \cap t A \neq \varnothing$ для любого элемента $a$ кольца $A$ и каждого элемента $t$ множества $T$. По условию либо сушествуют такие элементы $b_{1} \in A$ и $t_{1} \in T$, что $t b_{1}=$ $a t_{1}$, либо сушествуют такие элементы $b_{2} \in A$ и $t_{2} \in T$, что $a b_{2}=t t_{2}$. В первом случае $t b_{1}=a t_{1} \in a T \cap t A \neq \varnothing$. Допустим, что $a b_{2}=t t_{2} \in T$. Если $b_{2} \in T$, то $a b_{2} \in a T \cap$ $t A \neq \varnothing$. Допустим, что $b_{2} \in A \backslash T=N$. Так как $N$ - левьй идеал мультипликативной полугруппы $A$, то $a b_{2}=t t_{2} \in N \cap T=\varnothing$; получено противоречие. Поэтому $A$ имеет правое классическое кольцо частных $Q$.

Пусть $q_{1}$ и $q_{2}$ - элементы кольца $Q$. Существуют такие элементы $a_{1}, a_{2} \in A$ и $t \in T$, что $q_{1}=a_{1} t^{-1}$ и $q_{2}=a_{2} t^{-1}$. По условию либо существуют такие элементы $b_{1} \in A$ и $t_{1} \in T$, что $a_{1} b_{1}=a_{2} t_{1}$, либо существуют такие элементы $b_{2} \in A$ и $t_{2} \in T$, что $a_{2} b_{2}=a_{1} t_{2}$. В первом случае

$$
q_{2}=a_{2} t_{1} t_{1}^{-1} t^{-1} \in a_{1} b_{1} Q \subseteq q_{1} Q .
$$

Аналогично, $q_{1} \in q_{2} Q$ во втором случае. Поэтому $Q$ - цепное справа кольцо. В частности, кольцо $Q$ локально. По предложению $1 J(Q)=\bar{N}$ и $N$ - вполне первичньй идеал кольца $A$, совпадающий с множеством $A \cap J(Q)$. Так как $A$ - правьй порядок в цепном справа кольце $Q$, то непосредственно проверяется, то $A$ - равномерное справа кольцо.

Так как $A$ и $Q$ - равномерные справа кольца, то из леммы $3(1)$ следует, что $N_{\ell}$ - вполне первичньй идеал кольца $A$, совпадающий с $Z\left(A_{A}\right)$, и $\bar{N}_{\ell}-$ вполне первичньй идеал кольца $Q$, совпадающий с $Z\left(Q_{Q}\right)$. Поэтому $N_{\ell} Q \subseteq \bar{N}_{\ell}$. Пусть $s_{1}$ - элемент множества $\bar{N}_{\ell}$. Тогда $s_{1} s_{2}=0$ для некоторого ненулевого элемента $s_{2}$ кольца $Q$. Существуют такие элементы $c_{1}, c_{2} \in A$ и $u_{1}, u_{2} \in T$, что $s_{1}=c_{1} u_{1}^{-1}$ и $s_{2}=c_{2} u_{2}^{-1} \neq 0$. Существуют такие элементы $d \in A$ и $v \in T$, что $u_{1}^{-1} c_{2} u_{2}^{-1}=d v^{-1}$. Так как $s_{2} \neq 0$, то $d \neq 0$. Тогда $c_{1} d=c_{1} d v^{-1} v=s_{1} s_{2} v=0$ и $c_{1} \in N_{\ell}$. Поэтому

$$
\bar{N}_{\ell} \subseteq N_{\ell} Q \subseteq \bar{N}_{\ell}
$$

По лемме $4(7) \bar{N}_{r}$ - вполне первичньй идеал кольца $Q$.

Импликация $(3) \Rightarrow(1)$ очевидна.

Лемма 5. Пусть А- дистрибутивное справа кольио. Тогда верны следующие утверэдения:

(1) если $X$ и $Y$ - такие правье идеаль кольиа $A$, что $X \cap Y=0$, то

$$
(A X A) \cap(A Y A)=0, \quad(A X A)(A Y A)=(A Y A)(A X A)=0
$$

(следовательно, если $A$ - первичное кольцо, то $A$ - равномерное справа кольцо);

(2) если $B$ - вполне первичный левый идеал кольца $A$, то для любых әлементов $x \in A$ и $y \in A \backslash B$ сущ,ествуют такие әлементы $x^{\prime} \in A u y \in A \backslash B$, что $x y^{\prime}=y x^{\prime}$; 
(3) если $B$ - вполне первичный левый идеал кольиа $A$ и каждый әлемент множсества $A \backslash B$ регулярен в $A$, то $A \backslash B$-правое мнохсество Оре в $A$ и $A$-подкольцо некоторого чепного справа кольиа $Q$ являющегося правым кольцом частных относительно множества $A \backslash B$;

(4) если множество $N(A)$ всех нерегулярных әлементов кольиа $A$ является левым идеалом кольца $A$, то $A$ - правый порядок в иепном справа кольце;

(5) если $X$ - вполне первичный правый идеал кольца $A$, содержащийся в $J(A)$, то $X$ - идеал кольиа $A$ u $X=y X \subset y A$ для каждого әлемента $y \in A \backslash X$ (поэтому $X$ сравним по включению с любым правым идеалом кольца $A$ ).

ДоказАтельство. (1) По лемме $4(2)(A X A) \cap(A Y A)=0$. Возьмем любые элементы $z \in X$ и $y \in Y$. Обозначим через $x$ элемент $z y \in X$. Достаточно доказать, что $x=0$. Так как $x A \cap y A \subseteq X \cap Y=0$, то из леммы 2(1) следует, что существует такой элемент $a$ кольца $A$, что $x a=0$ и $y(1-a)=0$. Поэтому

$$
x=x a+x(1-a)=x(1-a)=z y(1-a)=0 .
$$

(2) По лемме 1 существуют такие элементы $a, c$ и $d$ кольца $A$, что $x a=y c$ и $y(1-a)=x d$. Если $a \in A \backslash B$, то можно положить $y^{\prime} \equiv a$ и $x^{\prime} \equiv c$. Поэтому можно считать, что $a \in B$. Тогда $1-a \in A \backslash B$ и $x d=y(1-a) \in A \backslash B$. Так как $B$ - левьй идеал, то $d \in A \backslash B$. В этом случае можно положить $y^{\prime} \equiv d$ и $x^{\prime} \equiv 1-a$.

(3) По (2) кольцо $A$ имеет правое кольцо частных $Q$ относительно множества $A \backslash B$. Остается доказать, что для любых элементов $q_{1}$ и $q_{2}$ кольца $Q$ верно, что либо $q_{1} \in q_{2} Q$, либо $q_{2} \in q_{1} Q$. Существуют такие элементы $x_{1}, x_{2} \in A$ и $t \in A \backslash B$, что $q_{1}=x_{1} t^{-1}$ и $q_{2}=x_{2} t^{-1}$. По лемме 1 существует такой элемент $a$ кольца $A$, что $x_{1} a \in x_{2} A$ и $x_{2}(1-a) \in x_{1} A$. Множество $A \backslash B$ содержит хотя бы один из элементов $a$ и $1-a$. Можно считать, что $a \in A \backslash B$. Тогда $a$ - обратимьй элемент кольца $Q$. Поэтому

$$
q_{1}=x_{1} t^{-1}=x_{1} a \cdot a^{-1} t^{-1} \in x_{2} A a^{-1} t^{-1} \subseteq x_{2} Q=q_{2} Q .
$$

(4) Это следует из (3) и того, что произведение любых двух регулярных элементов является регулярным элементом.

(5) По лемме $3(2) X$ - идеал кольца $A$. Пусть $y \in A \backslash X$. По лемме 1 существует такой элемент $z$ кольца $A$, что $x z \in y A$ и $y(1-z) \in x A \subseteq X$. Так как $X$ - вполне первичный правый идеал кольца $A$ и $y \in A \backslash X$, то $1-z \in X \subseteq J(A)$. Поэтому $z$ - обратимый элемент и $x=x z z^{-1} \in y A$. Тогда $x=y b$ для некоторого элемента $b$ кольца $A$. Так как $X$ - вполне первичньй идеал и $y \in A \backslash X$, то $b \in X$. Поэтому $x=y b \in y X$ и $X \subseteq y X \subseteq X$

ПРЕДЛОЖЕНИЕ 3. Пусть $A$ - дистрибутивное справа кольцо. Тогда равносильны следующие условия:

(1) любые два ненулевых идеала кольца $А$ имеют ненулевое пересечение;

(2) $A$ - равномерное справа кольио;

(3) $N_{r}(A)$ - подполугруппа аддитивной группы $A$;

(4) $N_{r}(A)$ - вполне первичный правый идеал кольиа $A, N_{\ell}(A)$ - вполне первичный идеал кольиа $A, N_{\ell}(A)$ совпадает с правым сингулярным идеалом $Z\left(A_{A}\right)$ кольца $A$ и либо $N_{r}(A)+N_{\ell}(A)=A$, либо правый идеал $N_{r}(A)$ и идеал $N_{\ell}(A)$ сравнимы по включению. 
ДоКАЗАТЕЛЬСТво. Эквивалентность условий (1) и (2) следует из леммы $5(1)$.

$(2) \Rightarrow(4)$ По лемме 4(6) $N_{r}(A)$ - вполне первичный правый идеал. Так как $A$ - равномерное справа кольцо, то каждый ненулевой правьй идеал кольца $A$ является существенным. Поэтому $N_{\ell}(A)$ совпадает с правым сингулярньг идеалом $Z\left(A_{A}\right)$ кольца $A$. $\mathrm{B}$ частности, $N_{\ell}(A)$ - идеал кольца $A$. Непосредственно проверяется, что $N_{\ell}(A)$ - вполне первичный идеал. По лемме $2(5)$ либо $N_{r}(A)+N_{\ell}(A)=A$, либо правый идеал $N_{r}(A)$ и идеал $N_{\ell}(A)$ сравнимы по включению.

Импликация $(4) \Rightarrow(3)$ очевидна.

Импликация $(3) \Rightarrow(2)$ следует из леммы 4(4).

Лемма 6. Пусть $A$ - такое дистрибутивное справа кольцо, что для каждого правого делителя нуля $a \in A$ әлемент $1-$ а регулярен в $A$. Тогда верны следующие утверәсдения:

(1) $A$ - равномерное справа кольцо и $N_{r}(A)+N_{\ell}(A) \neq A$;

(2) $N_{r}(A)$ и $N(A)$ - вполне первичные правые идеалы кольиа $A, N_{\ell}(A)$ - вполне первичный идеал кольиа $A$, правый идеал $N_{r}(A)$ и идеал $N_{\ell}(A)$ сравнимы по включению и либо $N(A)=N_{r}(A) \supseteq N_{\ell}(A)$, либо $N(A)-$ идеал кольца $A$ и $N(A)=$ $N_{\ell}(A) \supseteq N_{r}(A)$

(3) если $A N_{r}(A) \subseteq N(A)$, то $A$ - правый порядок в иепном справа кольце.

ДокАЗАтЕЛЬСтво. (1) Если $a \in N_{r}(A)$, то $1-a \notin N_{\ell}(A)$ по условию. Поэтому $N_{r}(A)+N_{\ell}(A) \neq A$.

Пусть $x$ - ненулевой элемент кольца $A$ и $y$ - такой элемент кольца $A$, что $x A \cap y A=0$. По лемме 2(1) существует такой элемент $a$ кольца $A$, что $x a=0$ и $y(1-a)=0$. Тогда $a \in N_{r}(A)$ и по условию $1-a$-регулярный элемент кольца $A$. Так как $y(1-a)=0$, то $y=0$. Поэтому $A-$ равномерное справа кольцо.

(2) Этот пункт следует из (1) и предложения 3.

(3) Так как $A N_{r}(A) \subseteq N(A)$, то из (2) следует, что $N(A)$ - вполне первичньй идеал. По лемме 5(4) $A$ - правьй порядок в цепном справа кольце.

ПРЕДЛОЖЕНИЕ 4. Пусть $A$ - дистрибутивное справа кольио. Тогда равносильны следующие условия:

(1) $A$ - правый порядок в чепном справа кольие $Q$;

(2) множество $N(A)$ является левым идеалом кольиа $A$;

(3) $N(A)$ и $N_{\ell}(A)$ - вполне первичные идеаль кольиа $A, N_{r}(A)$ - вполне первичный правый идеал кольиа $A$, правый идеал $N_{r}(A)$ и идеал $N_{\ell}(A)$ сравнимы по включению и либо $N(A)=N_{r}(A) \supseteq N_{\ell}(A)$, либо $N(A)=N_{\ell}(A) \supseteq N_{r}(A)$.

ДокАЗАТЕЛьство. $(1) \Rightarrow(2)$ Так как $Q$ - локальное кольцо, являющееся правым классическим кольцом частных кольца $A$, то $J(Q)$ является множеством всех необратимых элементов кольца $Q$ и $J(Q)$ совпадает с множеством $N(Q)$ всех нерегулярных элементов кольца $Q$. Пусть $x$ и $y$ - нерегулярные элементы кольца $A$ и $a$-элемент кольца $A$. Тогда $x, y \in N(A) \subseteq N(Q)=J(Q)$. Поэтому $x+y$ и $а x$ содержатся в $J(Q)$. Тогда элементы $x+y$ и $a x$ не обратимы в $Q$. Поэтому элементы $x+y$ и $a x$ не регулярны в $A$.

$(2) \Rightarrow(3)$ Так как сумма любых двух нерегулярных элементов кольца $A$ является нерегулярньм элементом, то для каждого правого делителя нуля $a$ кольца $A$ элемент $1-a$ регулярен в $A$. Поэтому утверждение следует из леммы 6(2). 
Импликация $(3) \Rightarrow(1)$ следует из леммы $6(3)$.

ПРЕДЛОЖЕНИЕ 5. Пусть $A$ - такое дистрибутивное справа кольио, что множество $N_{r}(A)$ содержится в $J(A)$. Тогда $A$ - правый порядок в иепном справа кольие $Q$, все множества $N(A), N_{r}(A)$ и $N_{\ell}(A)$ являются вполне первичными идеалами кольиа $A, N_{r}(A)=y N_{r}(A) \subset y A$ для каждого әлемента $y \in A \backslash N_{r}(A)$, идеаль $N_{r}(A)$ и $N_{\ell}(A)$ сравнимы по включению и либо $N(A)=N_{r}(A) \supseteq N_{\ell}(A)$, либо $N(A)=N_{\ell}(A) \supseteq N_{r}(A)$.

ДокАЗАТЕЛЬСТво. По лемме 6(2) $N_{r}(A)$ - вполне первичный правьй идеал кольца $A$. Кроме того, $N_{r}(A) \subseteq J(A)$ по условию. По лемме $5(5) N_{r}(A)$ - идеал кольца $A$ и $X=y X \subset y A$ для каждого элемента $y \in A \backslash X$. По лемме 6(3) $A$ - правый порядок в цепном справа кольце $Q$. Оставшиеся утверждения следуют из предложения 4 .

ОКОНЧАНИЕ ДОКАЗАТЕЛЬСТВ ТЕОРЕМ 1-3. Теорема 1 следует из предложения 2. Теорема 2 следует из предложения 4. Теорема 3 следует из предложения 5.

\section{СПИСОК ЦИТИРОВАННОЙ ЛИТЕРАТУРЫ}

[1] Dubrovin N. I. The Rational Closure of Group Rings of Leftordered Groups. Gerhard Mercator Universität Duisburg Gesamthochschule, 1994.

[2] Bessenrodt K., Brungs H. H., Törner G. Right Chain Rings. Part 1. Schriftenreihe des Fachbereich Mathematik. Universität Duisburg, 1990.

[3] Bessenrodt K., Brungs H. H., Törner G. Right Chain Rings. Parts 2a и 2b. Schriftenreihe des Fachbereich Mathematik. Universität Duisburg, 1992.

[4] Дубровин Н. И. Цепные области // Вестн. МГУ. Сер. матем., мех. 1980. № 1. С. 51-54.

[5] Дубровин Н. И. О цепных кольцах // УМН. 1982. Т. 37. № 4. С. 139-140.

[6] Behrens E. A. Ring Theory. New York: Academic Press, 1972.

[7] Кон П. Свободные кольца и их связи. М.: Мир, 1975.

[8] Tuganbaev A. A. Semidistributive Modules and Rings. Dordrecht-Boston-London: Kluwer Academic Publishers, 1998.

[9] Tuganbaev A. A. Distributive Modules and Related Topics. Amsterdam: Gordon and Breach, 1999.

[10] Stephenson W. Modules whose lattice of submodules is distributive // Proc. London Math. Soc. 1974. V. 28. № 2. P. 291-310.

[11] Menzel W. Über den Untergruppenverband einer Abelschen Operatorgruppe. Teil II. Distributive und M-Verbande von Untergruppen einer Abelschen Operatorgruppe // Math. Z. 1960. V. 74. №1. P. 52-65.

Московский энергетический институт 